\title{
Desenvolvimento de um equipamento para a caracterização não-destrutiva dos módulos elásticos de materiais cerâmicos
}

\section{(Development of equipment for non-destructive characterization of elastic moduli of ceramic materials)}

\author{
A. H. A. Pereira ${ }^{1}$, M. Venet $^{2}$, T. Tonnesen ${ }^{3}$, J. A. Rodrigues ${ }^{1}$ \\ ${ }^{I}$ Grupo de Engenharia de Microestrutura de Materiais - GEMM, Departamento de Engenharia de Materiais \\ Universidade Federal de S. Carlos, Rod. Washington Luis, km 235, S. Carlos, SP, Brasil 13565-905 \\ ${ }^{2}$ GCFerr, DF, UFSCar, S. Carlos, SP, Brasil \\ ${ }^{3}$ GHI/RWTH, Aachen, Alemanha \\ ha@atcp.com.br
}

\begin{abstract}
Resumo
Foi desenvolvido um equipamento para a caracterização não-destrutiva dos módulos elásticos aplicável a cerâmicas, concretos e materiais frágeis baseado na norma ASTM E 1875-00. Pode-se medir com exatidão, praticidade e simultaneidade o módulo de Young, de cisalhamento e a razão de Poisson. O equipamento obtém, via espectroscopia de ressonância sônica, as freqüências naturais de vibração flexurais e torcionais do corpo de prova e calcula os módulos. O equipamento foi desenvolvido em três partes: suporte do corpo de prova, eletrônica e software. O suporte aplica as condições de contorno mecânicas e a excitação necessária, a eletrônica gera e capta os sinais e o software executa os cálculos. A validação foi realizada com ensaios inter-técnicas e interlaboratoriais de materiais refratários e de referência. Os resultados mostraram que o equipamento é mais versátil do que as opções atuais para a medida simultânea das três propriedades elásticas apresentadas acima.

Palavras-chave: módulos elásticos, razão de Poisson, ressonância de barras, cerâmicas.
\end{abstract}

\begin{abstract}
In this work was developed an equipment for non-destructive characterization of elastic moduli applicable to ceramics, concretes, and brittle materials based on ASTME 1875-00 standard. The Young's modulus, shear modulus and Poisson's ratio can be simultaneously and easily measured with accuracy applying the new equipment. The natural torsional and flexural vibration frequencies of the sample are measured through sonic resonance spectroscopy. This permits the calculation of the elastic moduli. The system was developed in three parts: the sample holder, the electronics and the software. The sample holder applies the mechanical boundary conditions and excitation. The electronics captures and generates the signals. Finally, the software performs the calculations. The validation was carried out with inter-laboratory and inter-technique tests of refractories and reference materials. The results showed that the equipment is more versatile than the current options to the simultaneous measurement of the elastic moduli.
\end{abstract}

Keywords: elastic moduli, Poisson's ratio, resonance of bars, ceramics.

\section{INTRODUÇÃO}

Caracterização dos módulos elásticos

Os módulos elásticos são propriedades fundamentais para o projeto, desenvolvimento e controle de qualidade de materiais. Ao mesmo tempo em que são sensíveis à composição, processamento e dano do material, possuem correlação com outras propriedades usualmente medidas com ensaios destrutivos, como por exemplo, a resistência mecânica [1]. Os módulos elásticos podem ser caracterizados por métodos quase-estáticos, métodos dinâmicos ou por ultra-som. Os métodos quase-estáticos ou isotérmicos são baseados em ensaios mecânicos monotônicos, e os dinâmicos ou adiabáticos, nas técnicas de ressonância. Os valores determinados pelos métodos dinâmicos são ligeiramente maiores que aqueles determinados pelos estáticos em um percentual típico de $0,5 \%$ para metais [2]. Porém, este percentual não é relevante por ser muito inferior à incerteza dos ensaios mecânicos. Dentre estes métodos, os dinâmicos estão consolidados como a alternativa mais prática para materiais frágeis, não-homogêneos e para caracterizações em função da temperatura [3].

Os métodos dinâmicos e suas variações

Nestes métodos, a caracterização é realizada através das freqüências de ressonância do corpo, que são intrinsecamente associadas aos módulos elásticos [4]. Os métodos dinâmicos 
possuem duas variações principais normalizadas, a técnica de ressonância de barras [5] e de excitação por impulso [6]. Diversos aparatos são descritos na literatura para as duas técnicas. Contudo, há uma carência de soluções mais avançadas que permitam a caracterização simultânea dos módulos e da razão de Poisson com praticidade [3].

\section{O método dinâmico da excitação por impulso}

Neste método, o corpo de prova é excitado com uma leve pancada e responde com vibrações em suas freqüências naturais de acordo com as condições de contorno impostas. A técnica de excitação por impulso foi promovida pelo equipamento Grindosonic desenvolvido na década de 70 e comercializado atualmente com os mesmos recursos e funcionalidades. $\mathrm{Na}$ década de 90 , possibilitado pelos computadores, surgiu a segunda geração de equipamentos para a técnica da excitação por impulso [7-9]. Lançando mão da transformada de Fourier, esta nova geração proporcionou um avanço expressivo ao resolver o problema da discriminação das freqüências de ressonância. Porém o foco foi mantido na medida do módulo de Young, permanecendo os demais em segundo plano.

\section{O método dinâmico de ressonância de barras}

O método de ressonância de barras é muito conhecido e intuitivo, contudo o menos utilizado. Neste método o corpo de prova é dependurado por dois fios enlaçados em regiões próximas aos pontos nodais do modo de vibração de interesse, ou apoiados em suportes [5]. Há uma variação em que se trabalha com as ressonâncias longitudinais, mas apenas para a medida do módulo de Young [10]. Apesar de conceitualmente simples, a varredura manual e a posterior análise para a identificação e correlação dos picos é um procedimento demorado que demanda experiência, razão pela qual esta técnica é pouco utilizada. O equipamento mais avançado para esta técnica foi desenvolvido com foco em medidas em altas temperaturas [11]. Este equipamentoé capaz de medir simultaneamente todos os módulos e conta com o auxílio de um software para a identificação das freqüências e cálculo dos módulos. Para operar em altas temperaturas, a solução foi enlaçar o corpo de prova com fios refratários de carbono, que impõe o limite de $50 \mathrm{~g}$ como peso máximo e a necessidade de se ter uma atmosfera inerte na câmara para evitar a oxidação do carbono. A necessidade de atmosfera controlada aumenta expressivamente os custos e o peso máximo de $50 \mathrm{~g}$ praticamente inviabiliza a caracterização de materiais com agregados, como é o caso de refratários. Uma barra de material desse tipo, à base de alumina, com 25 x 25 x $150 \mathrm{~mm}^{3}$, que são as dimensões típicas para os ensaios de flexão, pesa entre 250 e $370 \mathrm{~g}$, dependendo da sua densidade aparente.

Norma ASTM E 1875-00 - Escopo e corpos de prova

Esta norma abrange a determinação dos módulos elásticos dinâmicos de materiais elásticos pelo método de ressonância de barras utilizando as equações desenvolvidas por Pickett [13]. É especialmente aplicável a materiais elásticos, homogêneos e isotrópicos. Materiais contendo agregados, fibras ou defeitos podem ser testados, mas estas características devem ser consideradas na análise dos resultados. Normas adicionais (C215, C623, C747, C848, C1198 e C1259) devem ser consultadas e observadas, dependendo do material em análise. De acordo com a norma ASTM E 1875-00, as freqüências de ressonância devem ser medidas em corpos de prova preferencialmente no formato de barras de secção retangular, utilizando um atuador para excitar e um sensor para captar a resposta.

\section{Norma ASTM E 1875-00 - Cálculos}

A norma ASTM E 1875-00 sugere a utilização das equações e método desenvolvidos por Pickett [13], que levam em consideração apenas os modos fundamentais de vibração. Para o módulo de Young, E, expresso em unidades de $\mathrm{Pa}$, a seguinte equação é utilizada:

$$
\mathrm{E}=0,9465\left(\frac{\mathrm{mf}_{\mathrm{f}}^{2}}{\mathrm{~b}}\right)\left(\frac{\mathrm{L}^{3}}{\mathrm{t}^{3}}\right) \mathrm{T}
$$

em que $\mathrm{m}$ é a massa do corpo de prova (em $\mathrm{kg}$ ), $\mathrm{f}_{\mathrm{f}}$ é a freqüência de ressonância flexional fundamental $(\mathrm{em} \mathrm{Hz})$, $\mathrm{t}$ é a dimensão da seção transversal paralela à direção de vibração (em m), Lé o comprimento da barra (em m), b é a largura da barra (em m) e T é um fator de correção de Pickett para o modo fundamental. No caso de barras de secção retangular, $\mathrm{T}$, que denota o efeito da deformação cisalhante $\mathrm{e}$ a inércia rotacional sobre $\mathrm{f}_{\mathrm{f}}$, é expresso como:

$$
\mathrm{T}=\mathrm{T}_{1}-\mathrm{T}_{2}-\mathrm{T}_{3}
$$

sendo

$$
\begin{aligned}
& \mathrm{T}_{1}=1+6,585\left(1+0,0752 v+0,809 v^{2}\right)\left(\frac{\mathrm{t}}{\mathrm{L}}\right)^{2} \\
& \mathrm{~T}_{2}=0,086\left(\frac{\mathrm{t}}{\mathrm{L}}\right)^{4}
\end{aligned}
$$

e

$$
\mathrm{T}_{3}=\frac{8,340\left(1+0,2023 v+2,173 v^{4}\right)\left(\frac{\mathrm{t}}{\mathrm{L}}\right)^{4}}{1+6,338\left(1+0,14081 v+1,536 v^{2}\right)\left(\frac{\mathrm{t}}{\mathrm{L}}\right)^{2}}
$$

em que $v$ é a razão de Poisson. A equação A é precisa para barras longas e finas, porém o erro aumenta conforme aumenta a razão $\mathrm{t} / \mathrm{L}$. Para amostras curtas e grossas, mas com $\mathrm{t} / \mathrm{L} \leq 0,4$, o erro estimado é de aproximadamente $1 \%$.

$\mathrm{O}$ módulo de cisalhamento (em Pa) é calculado pela seguinte equação: 


$$
\mathrm{G}=\frac{4 \mathrm{Lmf}_{\mathrm{f}}^{2}}{\mathrm{bt}}[\mathrm{B} /(1+\mathrm{A})]
$$

em que $f_{t}$ é a freqüência de ressonância torcional fundamental (em Hz). A e B são fatores de correção expressos como:

$$
\begin{aligned}
\mathrm{B} & =\left[\frac{\mathrm{b} / \mathrm{t}+\mathrm{t} / \mathrm{b}}{4(\mathrm{t} / \mathrm{b})-2,52(\mathrm{t} / \mathrm{b})^{2}+0,21(\mathrm{t} / \mathrm{b})^{6}}\right] \\
\mathrm{A} & =\left[\frac{0,52062-0,8776(\mathrm{~b} / \mathrm{t})+0,3504(\mathrm{~b} / \mathrm{t})^{2}-00078(\mathrm{~b} / \mathrm{t})^{3}}{12,03(\mathrm{~b} / \mathrm{t})+9,892(\mathrm{~b} / \mathrm{t})^{2}}\right]
\end{aligned}
$$

A razão de Poisson é calculada de forma iterativa pela seguinte equação

$$
v=\left(\frac{E}{2 G}-1\right)
$$

Desta forma, os valores de E e G são refinados também.

\section{Proposta deste trabalho}

A proposta deste trabalho foi desenvolver um equipamento mais versátil que os atuais para a caracterização simultânea do módulo de Young (E), de cisalhamento (G) e da razão de Poisson (v) pela técnica de ressonância de barras, de acordo com a norma ASTM E 1875-00. Para atingir este objetivo, a estratégia foi desenvolver uma eletrônica integrada e inovar na forma de suportar e excitar o corpo de prova, tendo como ponto de partida um arranjo experimental desenvolvido anteriormente no GEMM, UFSCar, baseado na integração de um conjunto de equipamentos e acessórios [12].

\section{EXPERIMENTAL}

\section{Suporte do corpo de prova}

Pensou-se num suporte no qual os apoios possuem a largura mínima necessária para promover estabilidade, e ao mesmo tempo, não inibir os modos flexionais. O sensor e o atuador entram em contato com o corpo de prova em posições que permitem a excitação tanto dos modos flexionais como dos torcionais. A posição ótima encontrada para os apoios foi em $0,25 \mathrm{~L}$, centrado com relação à largura, e os contatos do sensor e do atuador contrapostos em $0,32 \mathrm{~L}$. A posição ótima para o apoio usualmente descrita na literatura é no ponto nodal do modo flexional fundamental que ocorre em 0,224 $\mathrm{L}$, porém esta posição não permite a ocorrência simultânea $\mathrm{e}$ equilibrada dos modos torcionais e flexionais. O sensor e o atuador entram em contato com o corpo de prova através de varetas refratárias que podem operar até $1700^{\circ} \mathrm{C}$. No GEMM há um forno desenvolvido especialmente para medidas de módulos elásticos em função da temperatura que permite a inserção destas varetas [12].

\section{Eletrônica}

A sugestão da norma E 1875-00 é utilizar um arranjo de equipamentos: gerador de sinais, amplificador de potência e de sinais, freqüencímetro e osciloscópio. Todos estes equipamentos formam um complexo formidável que tem dificultado o uso pleno da técnica. Neste trabalho, a eletrônica foi desenvolvida integrando todas estas funções em blocos de um único equipamento. Ao invés do gerador de sinais, foi utilizado um sintetizador digital; do amplificador de potência, um circuito híbrido; do freqüencímetro, um contador; do amplificador de sinais, amplificadores operacionais de ganho programável e no lugar do osciloscópio, conversores analógicos digitais. Gerenciando todos estes blocos e componentes, um microprocessador com software embarcado executa a tarefa como um todo.

\section{Software}

Além do suporte e da eletrônica integrada, foi desenvolvido um software que facilita a identificação das freqüências e a execução dos cálculos. A partir da informação das características da amostra, respectivas tolerâncias e da faixa de freqüência desejada, o software comanda a eletrônica para que esta realize a varredura e obtenha, assim, o espectro. Obtido o espectro e a partir de um critério de amplitude mínima ajustada pelo usuário, o software lista os picos de ressonância, analisa as razões entre as freqüências, sugere uma seleção de picos e calcula os módulos e a razão de Poisson de acordo com as equações sugeridas pela norma, informando, inclusive, o intervalo de confiança dos resultados. A seleção dos picos pode ser alterada manualmente. Com a realização dos cálculos, o software gera um relatório dos resultados contendo todas as informações solicitadas pela norma.

\section{RESULTADOS E DISCUSSÃO}

$\mathrm{O}$ equipamento foi validado caracterizando-se diferentes materiais refratários e uma barra de aço com as técnicas de excitação por impulso, ressonância longitudinal e pulsoeco, disponíveis no instituto alemão GHI/RWTH-Aachen. Para as caracterizações via excitação por impulso, foi utilizado um equipamento Grindosonic Mk3, via pulso-eco, um equipamento Pundit-3 e via ressonância longitudinal, um arranjo experimental composto por um gerador de freqüências Philips PM-5191 e um osciloscópio LG OS3060D. 
Na Fig. 1 são apresentados os resultados para E das caracterizações inter-técnicas. Na Tabela I é apresentada a diferença média dos resultados das diversas técnicas com relação ao do equipamento desenvolvido; um valor negativo nesta diferença significa que a referida técnica apresentou um valor inferior ao do equipamento desenvolvido. Na Tabela II são mostrados os valores da literatura [14] e os valores medidos neste trabalho para a barra de aço. Entre parênteses está a diferença entre os valores encontrados com relação aos valores da literatura. Em média, o resultado obtido com o equipamento desenvolvido neste trabalho foi praticamente o mesmo que o obtido com a ressonância longitudinal,

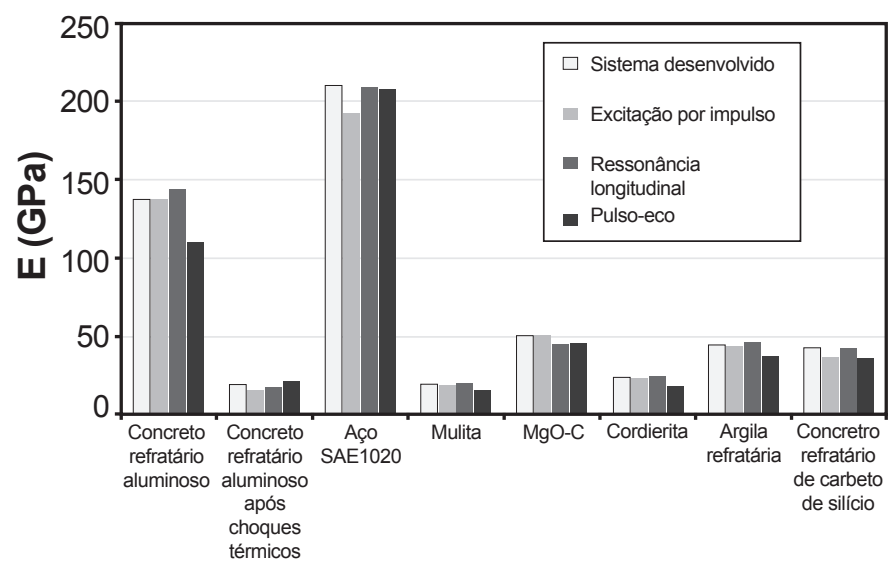

Figura 1: Resultados medidos com diferentes equipamentos para o módulo de Young.

[Figure 1: Results obtained with the developed and reference equipments for the Young's modulus.]

Tabela I - Diferença dos valores obtidos com as técnicas usuais com relação ao equipamento desenvolvido.

[Table I - Difference between the references equipments regarding the developed one.]

\begin{tabular}{cccc}
\hline & $\begin{array}{c}\text { Excitação } \\
\text { por impulso }\end{array}$ & $\begin{array}{c}\text { Ressonância } \\
\text { longitudinal }\end{array}$ & Pulso-eco \\
\hline $\begin{array}{c}\text { Diferença } \\
\text { média }\end{array}$ & $-5,58 \%$ & $-0,15 \%$ & $-12,2 \%$ \\
$\begin{array}{c}\text { Desvio padrão } \\
\text { da diferença }\end{array}$ & $6,99 \%$ & $4,63 \%$ & $12,1 \%$ \\
\hline
\end{tabular}

apenas $0,15 \%$ superior (Tabela I). Para a barra de aço, houve concordância entre o equipamento desenvolvido e as técnicas de ressonância longitudinal e pulso-eco (Tabela II). Porém, esta concordância não se repetiu na caracterização dos outros materiais com relação à técnica de pulso-eco (Tabela I). O problema é que, na técnica de pulso-eco, a razão de Poisson é importante nos cálculos. Porém, a mesma é estimada em 0,25 porque não é possível calculá-la somente a partir da velocidade longitudinal do som, seria necessário conhecer também a velocidade transversal. A velocidade longitudinal é a única possível de ser medida com os equipamentos usuais de pulso-eco sem a necessidade de utilização de transdutores e procedimentos especiais.

$\mathrm{Na}$ ressonância longitudinal também não é possível calcular a razão de Poisson, porém sua influência é bem menor. Por exemplo, ao se calcular os módulos de um material com razão de Poisson verdadeira de aproximadamente 0,15 (ex. cordierita), mas considerando-se nos cálculos o valor de 0,25 , o erro será da ordem de $0,5 \%$ para a ressonância longitudinal e de $5 \%$ para o pulso-eco. Para o equipamento desenvolvido, a razão de Poisson também é importante nos cálculos, mas ela é calculada e não estimada. Por isso, pode-se creditar a diferença entre os valores médios encontrados entre as técnicas de pulso-eco e do equipamento desenvolvido à relativa imprecisão da técnica do pulso-eco. No caso do aço, esta imprecisão não se manifesta porque a razão de Poisson estimada $(0,25)$ é próxima da real $(0,28)$.

A técnica de excitação por impulso levou a um resultado $6,06 \%$ abaixo dos indicados pela literatura para a amostra de aço, que é um material bem conhecido, bem comportado e fácil de ser medido. Esta diferença praticamente se repetiu na média para os outros materiais $(-5,58 \%)$. Não se identificou uma razão evidente e conclusiva para este deslocamento, que pode ser conseqüente da interferência de modos harmônicos ou mesmo descalibração do equipamento de excitação por impulso.

O módulo de cisalhamento e a razão de Poisson foram medidos apenas pelo equipamento desenvolvido neste trabalho e com uma diferença de $\pm 3 \%$ com relação aos valores da literatura para o aço (Tabela II). Não foi possível medir estes parâmetros com a técnica de ressonância longitudinal e pulso-eco, que possuía transdutores e acessórios apenas para a medida da velocidade longitudinal do som. Para a técnica de excitação por impulso, não havia a disponibilidade do

Tabela II - Valores de E, G e v, determinados para a barra de aço SAE 1020 com o equipamento desenvolvido e com os outros equipamentos comparados com valores da literatura [14].

[Table II - Comparison of the SAE 1020 steel literature values [14] for E, G e v with the obtained ones with the developed and reference equipments.]

\begin{tabular}{cccccc}
\hline & Literatura & $\begin{array}{c}\text { Equipamento } \\
\text { desenvolvido }\end{array}$ & $\begin{array}{c}\text { Excitação por } \\
\text { impulso }\end{array}$ & $\begin{array}{c}\text { Ressonância } \\
\text { longitudinal }\end{array}$ & Pulso-eco \\
\hline $\mathrm{E}(\mathrm{GPa})$ & 205 & $210,50(+2,68 \%)$ & $192,58(-6,06 \%)$ & $208,16(+1,54 \%)$ & $207,67(+1,30 \%)$ \\
$\mathrm{G}(\mathrm{GPa})$ & 80 & $82,94(+3,68 \%)$ & $\mathrm{X}$ & $\mathrm{X}$ & $\mathrm{X}$ \\
$v$ & 0,28 & $0,27(-3,57 \%)$ & $\mathrm{X}$ & $\mathrm{X}$ & $\mathrm{X}$ \\
\hline
\end{tabular}


acessório adequado para a medição dos modos torcionais.

Cabe destacar que além das freqüências fundamentais, o equipamento desenvolvido neste trabalho se mostrou capaz de obter o primeiro e o segundo harmônico tanto do modo flexional quanto do torcional (6 modos de vibração no total), criando a possibilidade da programação de algoritmos mais avançados e automatizados para o cálculo dos módulos.

\section{Praticidade do equipamento}

Comparado às demais técnicas pode-se dizer que o equipamento desenvolvido neste trabalho é mais versátil, pois é o único que calcula $E, G$ e $v$ simultaneamente e com precisão para diferentes tamanhos e pesos de corpos de prova sem a necessidade de acessórios ou procedimentos complexos. Em termos de rapidez de teste, o equipamento desenvolvido leva desvantagem com relação à excitação por impulso, que é muito rápida. Porém, facilita a análise dos resultados, cálculos e geração de relatórios com os recursos do software a ele associado. A técnica de pulso-eco também é rápida, porém imprecisa devido à importância da razão de Poisson para os cálculos e à impossibilidade de medi-la. A técnica da ressonância longitudinal é rápida e pode ser prática, desde que se tenha uma idéia da velocidade do som no material para a estimativa da freqüência de ressonância, o que muitas vezes não é o caso. A técnica de pulso-eco combinada com a ressonância longitudinal forma um bom conjunto: com o pulso-eco estima-se a ressonância longitudinal fundamental e com esta calcula-se com precisão o módulo de Young. Porém, este arranjo compromete a praticidade e a viabilidade financeira, porque em vez de uma, são duas técnicas, dois arranjos experimentais e dois treinamentos para os usuários.

\section{CONCLUSÕES}

Foi atingido com sucesso o objetivo de se desenvolver um equipamento mais versátil que os atuais para a caracterização simultânea dos módulos elásticos $(\mathrm{E}, \mathrm{G}$ e $v)$ pela técnica de ressonância de barras de acordo com a norma ASTM E 187500. O equipamento desenvolvido apresentou resultados coerentes com as técnicas e equipamentos comerciais bem estabelecidos, tanto na caracterização de materiais com microestrutura grosseira, como concretos refratários, quanto de materiais bem conhecidos e comportados, como o aço SAE 1020. O excelente desempenho do suporte de corpo de prova permitiu o registro tanto do modo flexional, como do torcional, até o $2^{\circ}$ harmônico (três primeiras freqüências de ressonância para cada um dos modos). Os próximos passos serão a automação do tratamento dos dados, a utilização de algoritmos mais avançados para os cálculos dos módulos e a integração do equipamento ao forno adaptado já existente no GEMM [12] para caracterizações em altas temperaturas.

\section{AGRADECIMENTOS}

Ao CNPq (Procs. 301073/2006-6 e 141868/2008-2),
CAPES (PROBRAL 258/07 e BEX 3926/08-7), FINEP (Proj. 4028-6) e DAAD (Deutscher Akademischer Austausch Dienst) pelos apoios financeiros.

\section{REFERÊNCIAS}

[1] F. Aly, C. E. Semler, Prediction of refractory strength using nondestructive sonic measurements, Am. Ceram. Soc. Bull. 64 (1985) 1555-58.

[2] R. Morrel, Measuring elastic properties of advanced technical ceramics - A review, UK National Physical Laboratory Report 42 (1996).

[3] R. Morrel, NPL measurement good practice guide No. 98 - Elastic module measurement, UK National Physical Laboratory Report (2006).

[4] S. Spinner, W. E. Tefft, A method for determining mechanical resonance frequencies and for calculating elastic moduli from these frequencies, ASTM Proceedings 61 (1961).

[5] ASTM E 1875-00, Standard test method of dynamic Young's modulus, shear modulus, and Poisson's ratio by sonic resonance, ASTM Standard approved October 10, 2000, published January 2007.

[6] ASTM E 1876-07, Standard test method for dynamic Young's modulus, shear modulus, and Poisson's ratio by impulse excitation of vibration, ASTM Standard approved June 1, 2007, published June 2007.

[7] G. Roebben, B. Bollen, A. Brebels, J. Humbeeck, O. Biest, Impulse excitation apparatus to measure resonant frequencies, elastic moduli, and internal friction at room and high temperature, Rev. Sci. Instruments 12, 68 (1997) 4511-15.

[8] P. A. Bosomworth, An exciting technique for quality analysis, Ceram. Ind. (2005) 20-24.

[9] C. Allaire, J. Allaire, A. Carbonneau, Room and high temperature measurement of the elastic properties of refractories using a new apparatus and wet-up, Light Metals - The Minerals, Metals Mater. Soc. Proc. (2004) 629-636.

[10] Erudite MkIV, CNS Farnell Limited, Commercial folder, Hertfordshire, England (2004).

[11] W. Lins, G. Kaindl, H. Peterlik, K. Kromp, A novel resonant beam technique to determine the elastic moduli in dependence on orientation and temperature up to $2000{ }^{\circ} \mathrm{C}$, Rev. Sci. Instr. 70, 7 (1999) 3052-58.

[12] A. E. M. Paiva, J. A. Rodrigues, Projeto e construção de um equipamento para a determinação do módulo elástico pelo método da ressonância de barras, Anais $45^{\circ}$ Cong. Bras. Cerâmica, S. Paulo, SP (2001) 301-313.

[13] G. Pickett, Equations for computing elastic constants from flexional and torsional resonant frequencies of vibration of prisms and cylinders, Am. Soc. Test. Mater. Proc. 45 (1945) 846-865.

[14] Jiangsu Tiancheng Steel Pipe Co., SAE 1020 Electronic data sheet [S.l.: s.n.], disponível em $<$ http://www.steel-tube. com/>, acesso em 08 dez. 2008.

(Rec. 17/06/2009, Ac. 28/08/09) 